\title{
Neue Möglichkeiten in der retinalen Diagnostik mittels OCT-Angiografie
}

\author{
New Possibilities in Retinal Diagnostics \\ Using OCT Angiography
}

Autoren

Institut

\author{
G. E. Lang, C. Enders, J. U. Werner
}

Augenklinik, Universitätsklinikum Ulm
Schlüsselwörter

- OCT-Angiografie

- altersbezogene Makuladegeneration

- diabetische Retinopathie

- retinaler Venenverschluss

- retinaler Arterienverschluss

Key words

- OCT angiography

- age-related macular degeneration

- diabetic retinopathy

- retinal vein occlusion

- retinal artery occlusion

eingereicht 26.2.2016 akzeptiert 4.3.2016

\section{Bibliografie}

Dol http://dx.doi.org/ 10.1055/s-0042-105325 Klin Monatsbl Augenheilkd 2016; 233: 613-621 @ Georg Thieme Verlag KG Stuttgart New York · ISSN 0023-2165

\section{Korrespondenzadresse}

Prof. Gabriele E. Lang

Augenklinik

Universitätsklinikum Ulm

Prittwitzstr. 43

89075 Ulm

Tel.: + 49/(0)731/500-59001

Fax: $+49 /(0) 731 / 500-59002$

gabriele.lang@uniklinik-ulm.de

\section{License terms}

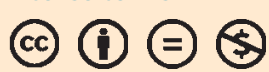

\section{Zusammenfassung}

$\nabla$

Hintergrund: Seit Kurzem sind Geräte zur Anwendung der OCT-Angiografie (OCTA) im klinischen Alltag verfügbar. Ziel dieser Arbeit ist es, Möglichkeiten, Vorzüge und Grenzen der OCTA zur Diagnose von Erkrankungen des Hinterabschnitts in der klinischen Anwendung zu beschreiben.

Patienten/Methoden: Beispielhaft werden Patienten mit diabetischer Retinopathie, retinalen Gefäßverschlüssen und altersbedingter Makuladegeneration dargestellt, bei denen zusätzlich zur Fluoresceinangiografie (FA) auch eine OCTAUntersuchung durchgeführt wurde. Dazu wurden mit der neuen AngioPlex ${ }^{\mathrm{TM}}$-OCTA-Technologie $6 \times 6 \mathrm{~mm}$-Volumenscans um die Fovea aufgenommen. Sofern erforderlich, wurden $3 \times 3 \mathrm{~mm}$-Volumenscans im Bereich besonders interessanter Areale erstellt. Die Befunde aus FA und OCTA wurden korreliert und vergleichend beurteilt.

Ergebnisse: Die OCTA ließ sich in wenigen Sekunden durchführen, war gut in die Standarduntersuchungen zur Diagnose von Erkrankungen der Retina und Aderhaut integrierbar und lieferte sehr detailreiche, 3-dimensionale Darstellungen des gesamten Mikrogefäßsystems von Netz- und Aderhaut. Alle mikrovaskulären Veränderungen wie Mikroaneurysmen, intraretinale mikrovaskuläre Anomalien, nicht perfundierte Areale, Veränderung der foveal-avaskulären Zone (FAZ) und Neovaskularisationen konnten zuverlässig dargestellt werden. Insgesamt zeigte sich eine gute Übereinstimmung mit den Ergebnissen der FA. Zusätzlich lieferte die OCTA detailreiche und tiefenselektive Informationen, die eine Beurteilung des räumlichen Verlaufs der Gefäße ermöglichten. Aufgrund des begrenzten Scanausschnitts war es zur Erfassung von Gefäßveränderungen in der mittleren Peripherie mittels OCTA erforderlich, deren Lage vorher in der FA zu bestimmen. Schrankenstörungen der Gefäße sind in der OCTA

\section{Abstract \\ $\nabla$}

Background: Instruments for using OCT angiography (OCTA) in daily clinical practice have recently become available. The aim of this paper is to report the possibilities, advantages and limitations of OCTA in the clinical diagnosis of diseases of the posterior segment of the eye.

Patients/Methods: Patients with diabetic retinopathy, retinal vascular occlusions, and age-related macular degeneration who had been assigned to fluorescein angiography (FA) additionally underwent an AngioPlex ${ }^{\mathrm{TM}}$-OCTA examination, which captures a $6 \times 6 \mathrm{~mm}$ scanning area centred on the fovea. If deemed necessary, $3 \times 3 \mathrm{~mm}$ volume scans were created in regions of interest. The findings of FA and OCTA were correlated and compared.

Results: The OCTA procedure took only a few seconds, was easily integrated into the standard OCT diagnostic imaging procedure, and delivered highly detailed, three dimensional images of the entire microvasculature of the retina and choroid. Microvascular changes, such as microaneurysms, intraretinal microvascular abnormalities, nonperfused areas, alterations in the foveal avascular zone (FAZ) and neovascularization were reliably detected. Overall, OCTA results were in good agreement with the results of the FA. Additionally, OCTA provided more detailed and three dimensional information and thus permitted a better assessment of the spatial extension of microvascular abnormalities. Due to OCTA's limited scanning area, vascular alterations in the mid-periphery were detected only if their location had already been determined by FA. Although OCTA does not show leakage, macular oedema can be diagnosed from OCTA, together with OCT thickness measurements.

Conclusion: OCTA provides important three dimensional information on vascular alterations and is already an indispensable diagnostic 
nicht darstellbar, dennoch konnten Makulaödeme mittels OCTA und korrespondierender OCT-Dickenmessung gut diagnostiziert werden.

Schlussfolgerung: Die OCTA liefert wichtige räumliche Informationen und ist schon heute eine unverzichtbare Untersuchungsmethode. Aufgrund ihres nicht invasiven Charakters und ihrer schnellen Durchführung ist sie gut als Monitoring-Methode geeignet und kann frühzeitige Diagnosestellungen ermöglichen. Prospektive klinische Studien sind erforderlich, um einheitliche Analysestrategien zu definieren. Die OCTA wird mit hoher Wahrscheinlichkeit die Diagnostik von Netzhaut und Aderhauterkrankungen revolutionieren, inwieweit sie jedoch die FA ersetzen wird, lässt sich derzeit noch nicht abschätzen. method. As the procedure takes just a few seconds and can be performed non-invasively, OCTA is well suited as a monitoring method and may allow early diagnosis. In this sense, prospective studies are required to determine precise OCTA analytical strategies for specific diseases. It is very likely that OCTA will revolutionise the diagnosis of retinal and choroidal diseases; however, it is not yet clear estimated to what extent it will replace FA.

\section{Einleitung \\ $\nabla$}

Mit der Einführung der OCT-Angiografie (OCTA) hat eine neue Ära in der OCT-Bildgebung und Retinadiagnostik begonnen. Erstmals kann nun auch die Mikrodurchblutung von Netzhaut und Aderhaut mittels nicht invasiver OCT-Untersuchung über die Darstellung perfundierter Gefäße anhand deren Flussregistrierung erfasst werden. Damit besteht die Möglichkeit, den Perfusionsstatus des hinteren Augenabschnitts in kurzer Zeit und im Gegensatz zum bisherigen Goldstandard Fluoresceinangiografie (FA) - auch ohne Farbstoffinjektion zu erheben. Zudem kann in der OCTA über die Flussregistrierung von sich bewegenden Erythrozyten ein 3-dimensionales Bild des Mikrogefäßsystems dargestellt werden, während bei der FA ein 2-dimensionales Summenbild vom Fluoreszenzverhalten der Netzhaut und Aderhaut erhoben wird.

Wesentliche Grundlagen dieser neuen Technologie sind schnellere Scangeschwindigkeiten der modernen OCT-Geräte sowie neue Auswertungsalgorithmen, die es ermöglichen, zeitliche Kontrastunterschiede an einem Ort zu detektieren. So können erstmals Kontrastunterschiede zwischen 2 rasch aufeinanderfolgenden B-Scans erfasst werden, die durch sich im Blutfluss bewegende Erythrozyten hervorgerufen werden. Um den Blutfluss mittels OCTA zu detektieren, wird zunächst jeder B-Scan eines Volumenscans an der exakt gleichen Position mehrfach kurz hintereinander wiederholt und die zeitlichen Kontrastunterschiede an dieser Position werden analysiert. Aus der vergleichenden Auswertung aller B-Scans eines Volumenscans ergeben sich neben Bereichen mit gleichbleibendem Kontrast auch Bereiche mit zeitlichen Kontrastunterschieden. Diese stellen einen Blutfluss dar, sodass das Gefäßsystem mittels OCTA innerhalb des vom Volumenscan erfassten Bereichs 3-dimensional dargestellt werden kann. Durch Segmentierung zwischen bestimmten Netzhautschichten können zudem partielle En-face-Darstellungen des Mikrogefäßsystems dieser Netzhautbereiche in beliebiger Tiefe erstellt werden.

Erste Geräte zur OCT-Angiografie sind nun verfügbar oder werden in Kürze auf den Markt kommen. Auch wenn die OCTA derzeit noch sehr neu ist und ihre klinische und wissenschaftliche Evaluierung gerade erst begonnen hat, so liegen doch bereits erste Ergebnisse zu ihrer Anwendung bei verschiedenen Erkrankungen des Hinterabschnitts vor [1-17].

Ziel dieser Arbeit ist es, die Möglichkeiten der klinischen Anwendung der OCTA aus aktuellen Erfahrungen mit der ersten von der FDA zugelassenen OCTA-Plattform, der neuen AngioPlex ${ }^{{ }^{\mathrm{TM}}}{ }_{-}$ OCTA-Technologie von Zeiss in Kombination mit dem CIRRUS HD-OCT 5000 (Carl Zeiss Meditec, Inc., Dublin, USA), zur Diagnose verschiedener Erkrankungen des Hinterabschnitts darzustellen. Dabei werden Vorzüge und Grenzen der Methode beschrieben.

\section{Methoden \\ $\nabla$}

Seit Kurzem sind OCT-Geräte für die Anwendung im klinischen Alltag erhältlich, mit denen eine OCTA durchgeführt werden kann. Die AngioPlex-OCTA ist seit Oktober 2015 für den klinischen Einsatz verfügbar. Seitdem haben wir erste klinische Erfahrung sammeln können, indem bei Patienten mit Erkrankungen des Hinterabschnitts zusätzlich zur FA gleichzeitig auch eine AngioPlex-OCTA-Untersuchung durchgeführt wurde. Die OCTABefunde wurden mit den FA-Befunden korreliert. Dazu wurden zunächst $6 \times 6 \mathrm{~mm}$-Volumenscans um die Fovea aufgenommen. Waren für bestimmte Areale detailliertere Aufnahmen erforderlich, so wurden zusätzlich $3 \times 3 \mathrm{~mm}$-Volumenscans um diese „Region of Interest" (ROI) erstellt.

Das CIRRUS HD-OCT 5000 arbeitet mit Scangeschwindigkeiten von bis zu $68 \mathrm{kHz}$, ist mit einem Zeilenscan-Ophthalmoskop (LSO) ausgestattet und stellt zudem durch das FastTrac ${ }^{\mathrm{TM}}$-EyeTracking-System sicher, dass auch kleine Augenbewegungen zuverlässig nachverfolgt werden. Dadurch wird gewährleistet, dass ein sehr kontrastreiches Fundusbild und eine bessere Überlagerung verschiedener Scans desselben Auges erzielt und Bewegungsartefakte reduziert werden können. Die zeitlichen Kontrastunterschiede zur Ermittlung des Blutflusses werden in der AngioPlex-Technologie mit Algorithmen des sog. „Optical Micro Angiography Complex“ (OMAG ${ }^{\mathrm{C}}$ ) erhoben. Diese werten im Gegensatz zu den meisten anderen Algorithmen zur Ermittlung zeitlicher Kontrastunterschiede sowohl Amplituden- als auch Phasensignale der B-Scans vergleichend aus. Dies trägt zu einer präzisen Identifikation zeitlicher Kontrastunterschiede und dementsprechend hoher Bildqualität bei. Wie eine aktuelle Studie nahelegt, in der verschiedene, derzeit in der OCTA verwendete Algorithmen verglichen wurden, können die besten Ergebnisse in der Visualisierung des retinalen mikrovaskulären Gefäßsystems mit OMAGC${ }^{\mathrm{C}}$-Algorithmen erreicht werden [18].

\section{Ergebnisse \\ $\nabla$}

Es wurden Patienten mit unterschiedlichen Erkrankungen des Hinterabschnitts mittels AngioPlex-OCTA untersucht. Im Folgenden werden typische, mit der neuen OCTA-Technologie erhobene Befunde beschrieben und die OCTA-Einsatzmöglichkeiten an- 


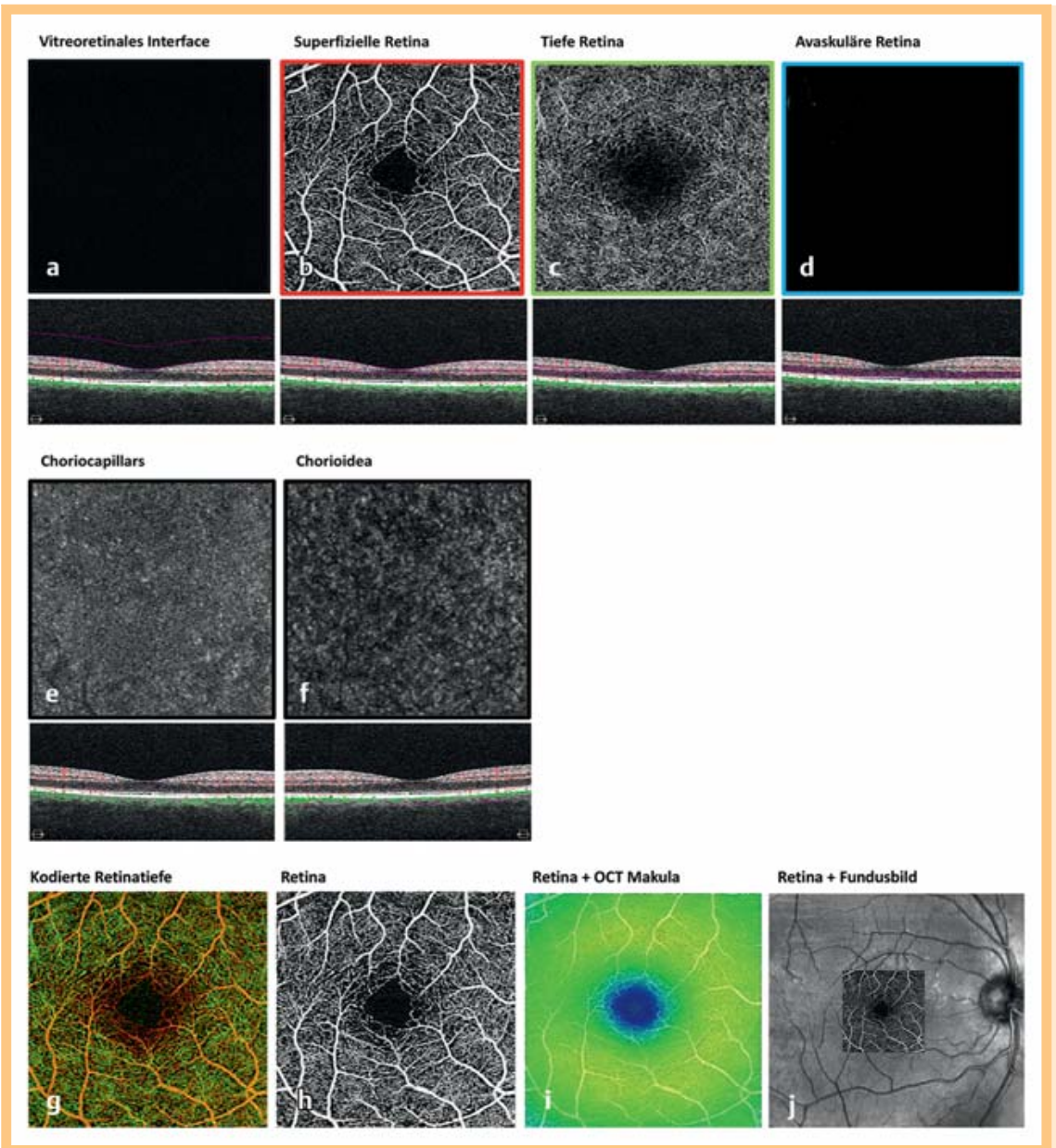

Abb. 1 Auge ohne Pathologie mit Darstellung des Bereichs. a Vitreoretinales Interface (der Bereich vor der Netzhaut zeigt normalerweise keine Gefäße und erscheint daher schwarz); b Gefäßdarstellung oberflächliche Retina; c Gefäßdarstellung tiefe Retina und $\mathbf{d}$ avaskuläre Retina (diese enthält normalerweise keine Gefäße und erscheint daher schwarz). Die unteren Abbildungen zeigen jeweils die En-face-Analyse im korrespondierenden B-ScanDurchlauf mit den Segmentierungslinien (dünne lila Linien; die roten Punkte zeigen die Flussregistrierung oberhalb des retinalen Pigmentepithels [RPE], die grünen Punkte die Flussregistrierung unterhalb des RPE); e Choriocapillaris und $\mathbf{f}$ Chorioidea jeweils mit der Darstellung der En-face-Analyse im korrespondierenden B-Scan-Durchlauf mit den Segmentierungslinien.

Auge ohne Pathologie mit Darstellung der Möglichkeiten der Datenanalyse der AngioPlex-Aufnahmen mit $\mathbf{g}$ farbcodierter Retinatiefe, $\mathbf{h}$ Gefäßen der gesamten Retina, i OCT-Angiografie überlagert mit OCT-Makuladicke und j OCT-Angiografie auf Fundusaufnahme projiziert.

hand von Fällen verschiedener Erkrankungen des Hinterabschnitts beispielhaft erläutert.

\section{Klinische Anwendung der}

\section{AngioPlex-OCTA-Untersuchung}

Die OCT-Angiografie lässt sich als Standarduntersuchung vergleichbar der bisherigen OCT-Untersuchung zur Diagnostik verschiedener Erkrankungen des Hinterabschnitts einfach anwenden. Die AngioPlex-OCTA mit dem CIRRUS HD-OCT 5000 gewährleistet über die „Single-Scan Simplicity“ der Plattform, dass mit nur einem zusätzlichen Scan OCTA-Darstellungen des Mikrogefäßnetzwerks im ausgewählten Scanbereich generiert werden. Die Aufnahmezeiten der OCTA-Messungen betragen bei guter Kooperation des Patienten nur 5-10 Sekunden pro Scan. Bei mangelnder Fixation des Patienten oder bei stark eingetrübten Medien (z. B. fortgeschrittene Katarakt oder starke Einblutungen in den Glaskörper oder die Retina) waren die OCT-Messungen teilweise beeinträchtigt - eventuell aufgrund der Eintrübung selbst oder auch infolge beeinträchtigter Kooperationsfähigkeit des Patienten aufgrund des herabgesetzten Visus. Insbesondere bei starken Glaskörper- oder Netzhautblutungen liefert allerdings auch die FA häufig nur unzureichende Bilder.

Bereits kurz nach dem Aufnahmevorgang stehen sowohl verschiedene Perfusions- als auch korrespondierende morphologische Analysen gleichzeitig auf dem Display zur Verfügung und ermöglichen vielfältige Auswertungen auf einen Blick. Über die AngioPlex-Software wird eine automatische retinale und choroidale Segmentierung vorgenommen, und verschiedene En-face-Bilder ausgewählter Netzhautschichten des gescannten Netzhautabschnitts werden voreingestellt angezeigt. ( $\bullet$ Abb. $\mathbf{1} \mathbf{a}-\mathbf{j}$ ). Einige dieser Analysedarstellungen sind farbcodiert, sodass eine schnelle Orientierung in der Netzhauttiefe erleichtert wird. So zeigen z.B. die „Superfizielle Retina“ (in Rot, $\boldsymbol{Q}$ Abb. 1 b) bzw. die „Tiefe Retina“" (in Grün, $\bigcirc$ Abb. 1 c) das oberflächliche bzw. das tiefe Mikrogefäßsystem der Netzhaut, während in der „Avaskulären Retina" (in Blau, $\mathbf{O}$ Abb. 1 d) der Bereich abgebildet wird, der bei gesunden Augen avaskulär erscheint. Dem korrespondierenden „B-Scan-Durchlauf“ kann jeweils entnommen werden, welche Segmentierung (feine lila Linien) zur Erstellung der jeweiligen En-face-Darstellung gewählt wurde. Zudem zeigen im „B-ScanDurchlauf" rote Punkte in den Netzhautschichten oberhalb des retinalen Pigmentepithels (RPE) und grüner Punkte in den Schichten unter dem RPE an, wie der erfasste Bereich perfundiert ist. Es werden also nur perfundierte ortsständige oder neovaskuläre Gefäße durch Flussregistrierung dargestellt. Durch Auswahl eigener Segmentierungslinien können auch anwenderbezogene En-face-Darstellungen klinisch besonders relevanter Schichten von der vitreoretinalen Grenzschicht bis zur Aderhaut erstellt werden. Hilfreich ist hierbei die „Film-Option“, mit welcher der gescannte Bereich in der gesamten Tiefe „abgefahren“ werden kann. So können auch Schichten, in denen nur geringe Veränderungen auftreten, schnell und zuverlässig identifiziert und selektiv zur Segmentierung ausgewählt werden.

Darüber hinaus erleichtern weitere, kombinierte Darstellungen der Datenanalysen eine umfassende Befundung: In der Darstellung „Retina“ werden die Informationen der eingangs beschrie- 


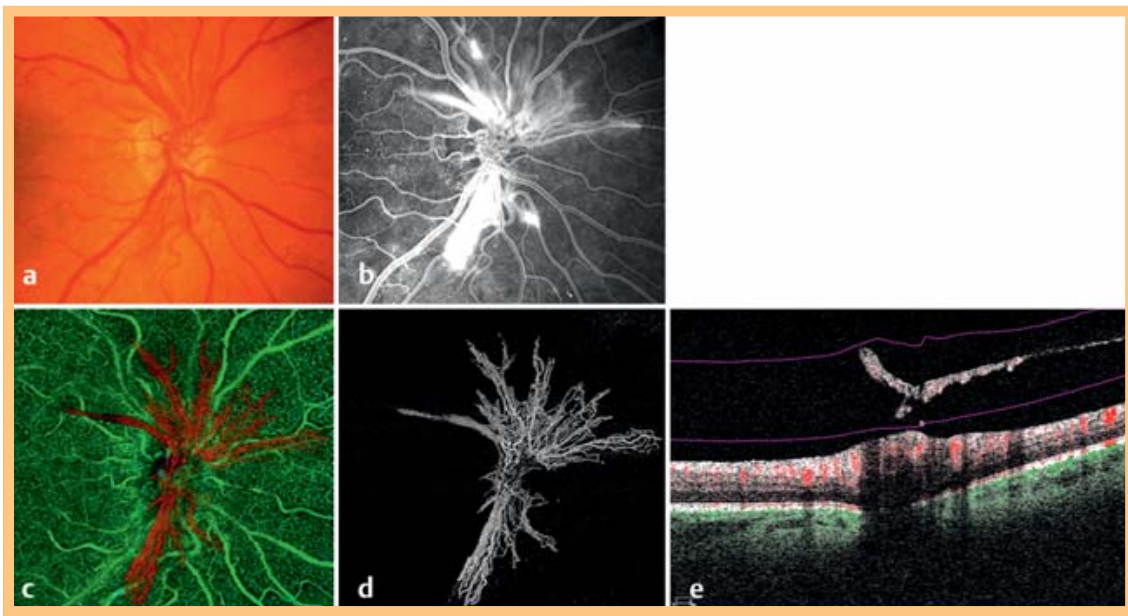

Abb. 2 Papilläre Neovaskularisation (NVD) bei diabetischer Retinopathie. a klinisches Bild; b Fluoresceinangiografie; $\mathbf{c}$ codierte Retinatiefe (NVD rot); d NVD in der Segmentierung vitreoretinales Interface; e B-Scan-Durchlauf und Segmentierungslinien (dünne lila Linien; die roten Punkte zeigen die Flussregistrierung der NVD und oberhalb des retinalen Pigmentepithels [RPE], die grünen Punkte die Flussregistrierung unterhalb des RPE).

benen, automatisch voreingestellten Ebenen der Netzhaut kombiniert dargestellt und so das gesamte Gefäßsystem der Retina als 3-dimensionales Bild räumlich visualisiert. In der Darstellung „codierte Retinatiefe“ werden die verschiedenen Ebenen zudem farbcodiert aufgeführt (ه Abb.1g). Dadurch wird der 3dimensionale Eindruck verstärkt, was die Tiefenorientierung erleichtert und hilft, den räumlichen Verlauf von Gefäßen rasch zu erkennen. So kann anhand dieser Darstellung häufig schon auf den ersten Blick erfasst werden, in welchen Schichten der Retina Veränderungen vorliegen. In der Darstellung „Retina + OCT Makula“ wird die OCTA-Analyse des gesamten erfassten retinalen Mikrogefäßsystems mit der OCT-Analyse zur Makuladicke überlagert ( Abb. 1 i). So können Bereiche mit einer durch Ödem veränderten Makuladicke rasch erkannt und ggf. mit vaskulären Veränderungen korreliert werden. Insbesondere anhand dieser Darstellung lässt sich häufig gut erkennen, wie der Scan positioniert werden muss, um eine klinisch relevante ROI präzise und detaillierter zu erfassen. Die Überlagerung des Mikrogefäßsystems der Netzhaut mit dem entsprechenden Fundusbild ( Abb. 1 j) ermöglicht einen komfortablen Abgleich mit der klinischen Befundung und trägt zur Verifizierung der Diagnose bei. Zudem lassen sich über das Display zusätzlich auch die entsprechenden Querschnitts- und En-face-Darstellungen der intensitätsbasierten OCT anwählen. Dadurch können auch strukturelle Veränderungen wie eine erhöhte Retinadicke, subretinale Flüssigkeit oder ein zystoides Makulaödem im Detail begutachtet und im Zusammenhang mit dem OCTA-Bild beurteilt werden.

\section{Diabetische Retinopathie (DR)}

In den OCTA-Aufnahmen bei DR zeigt sich grundsätzlich eine gute Übereinstimmung mit den Ergebnissen der FA. Dabei liefern die OCTA-Bilder im Vergleich zur FA detailreichere und vor allem zusätzliche, tiefenselektive Informationen. So konnten bei allen Patienten mit proliferativer DR mittels OCTA die Neovaskularisationen im Bereich innerhalb der Gefäßbögen bis zur Papille zuverlässig identifiziert werden, die auch in der FA detektiert wurden. Auch Neovaskularisationen in der mittleren Peripherie (d.h. außerhalb der Gefäßbögen), die mittels FA identifiziert wurden, konnten nachträglich in der OCTA präzise dargestellt werden. Dazu war es allerdings aufgrund des begrenzten Scanausschnitts der OCTA erforderlich, die Lage der Neovaskularisationen vorher in der FA zu bestimmen. Der Vorteil der zusätzlichen OCTA-Aufnahmen liegt dabei in ihrer detaillierten und 3-dimensionalen Darstellung des räumlichen Verlaufs der Neovaskularisationen, die anhand der 2-dimensionalen FA-Aufnahmen so nicht gewonnen werden können. Auch die Größe der Neovaskularisationen ist in der OCTA besser bestimmbar, da sie in der FA nur in der Frühphase erkennbar sind und dann durch die Fluoresceinextravasation, die auch an Ausdehnung zunimmt, maskiert werden. Auch kann die räumliche Ausdehnung in den Glaskörperraum mit der FA nicht sichtbar gemacht werden. Hier ist beispielhaft der Fall eines Patienten mit proliferativer DR dargestellt, bei dem mittels OCTA der räumliche Verlauf der papillären Neovaskularisation (NVD) bis in die vitreoretinale Grenzschicht hinein exakt verfolgt werden kann ( $\triangle$ Abb. 2 a-e). Im Fundusbild war die NVD erkennbar, die in der FA bestätigt wurde. Ausmaß und räumliche Lage der NVD ließen sich in der FA allerdings nicht so exakt bestimmen, wie es schließlich anhand der OCTA-Aufnahmen möglich war. So ist in der automatisch angezeigten OCTAAnalyse „codierte Retinatiefe“ aufgrund der roten Färbung der NVD ersichtlich, dass die NVD in oberflächlichen Schichten lokalisiert ist ( Abb. 2c). Ein „Abfahren“ der Netzhautschichten mittels „Film-Option“ zeigt zudem, dass die NVD in den vitreoretinalen Bereich proliferiert ist. Wie im „B-Scan-Durchlauf“ erkennbar, wurden daher zur exakten Visualisierung der präpapillär gelegenen NVD eigene Segmentierungslinien ober- und unterhalb der NVD gesetzt ( $\triangle$ Abb. 2e). So gelingt mittels AngioPlex-OCTA eine sehr präzise und detaillierte Darstellung der NVD im vitreoretinalen Interface, das im gesunden Auge in der OCTA-Aufnahme aufgrund seiner Gefäßfreiheit schwarz erscheint (vgl. @ Abb. 1 a und Abb. 2d). Ausmaß, räumlicher Verlauf und Struktur der Neovaskularisation stellen sich in der OCTA-Aufnahme deutlich detailreicher dar als in der FA, die keine 3-dimensionale Zuordnung erlaubt, und lassen sich daher präziser beurteilen. Die im „BScan-Durchlauf“ in der NVD sichtbaren roten Punkte zeigen zudem deren Durchblutung an.

In der OCTA sind perfundierte Mikroaneurysmen, mikrovaskuläre Anomalien, eine vergrößerte foveal avaskuläre Zone und nicht perfundierte Areale der Netzhaut gut darstellbar. Auch ein diabetisches Makulaödem (DMÖ) ist mittels OCTA und korrespondierender OCT-Dickenmessung gut und zuverlässig diagnostizierbar. Es lässt sich jedoch keine Schrankenstörung der Gefäße wie in der FA darstellen. Allerdings korrespondiert die Farbstoffleckage in der FA nicht unbedingt mit der Lage des Makulaödems. Wie der hier exemplarisch aufgeführte Fall zeigt ( Abb.3a-d), ermöglicht die Datenanalyse „Retina + OCT Makula“, in der die AngioPlex-Gefäßdarstellung mit der OCT-Makuladicke überlagert dargestellt wird, auf den ersten Blick die Lokalisation des 


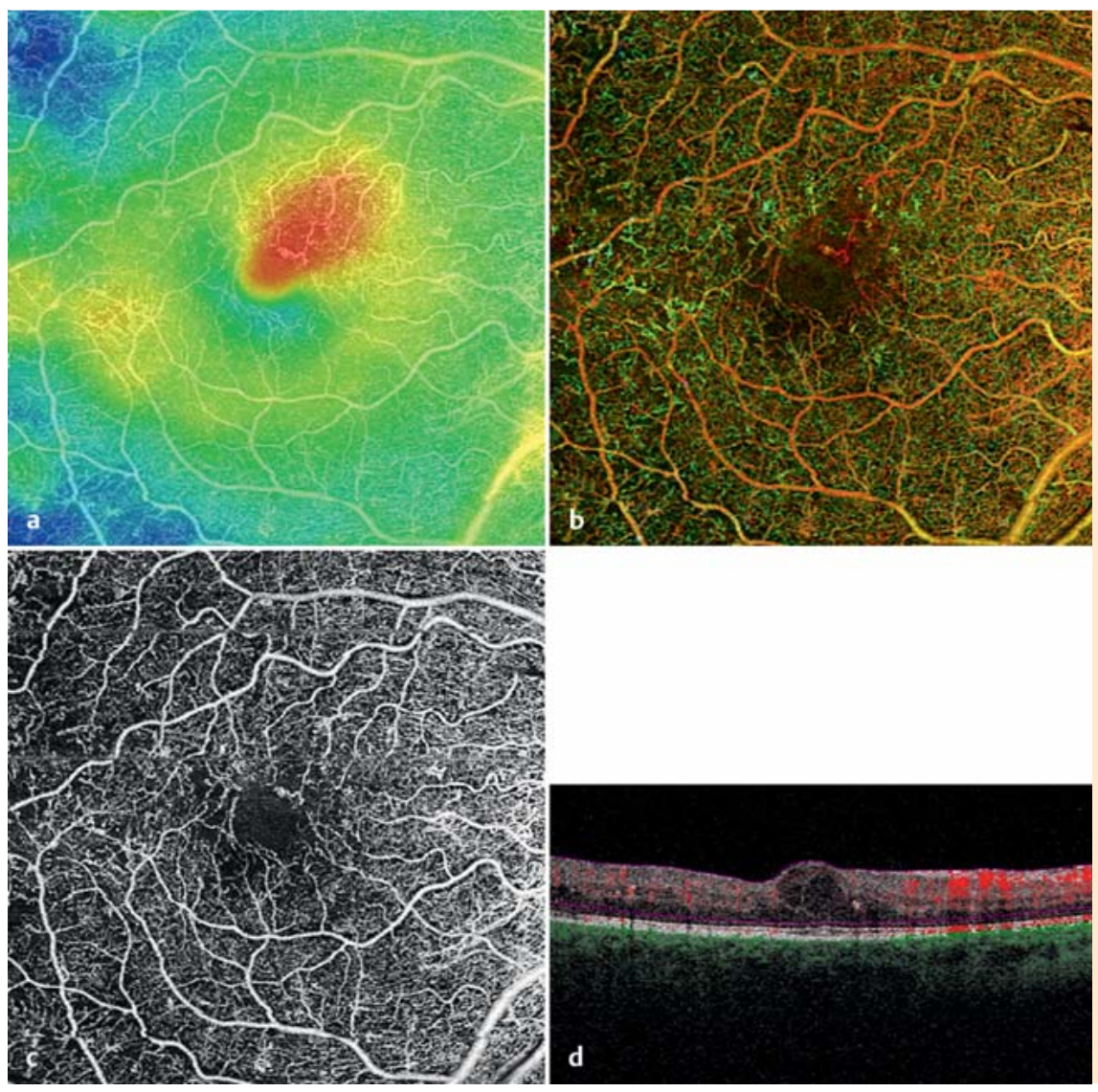

Abb. 3 Diabetisches Makulaödem. a OCT-Angiografie-Gefäßdarstellung überlagert mit OCT-Makuladicke (roter Bereich Makulaödem); b codierte Retinatiefe mit Mikroaneurysmen, kleinen nicht perfundierten Arealen und gering vergrößerter foveal-avaskulärer Zone; c Retinadarstellung oberflächliche und tiefe Schichten; d B-Scan-Durchlauf zeigt das Makulaödem und die Segmentierungslinien (dünne lila Linien; die roten Punkte zeigen die Flussregistrierung oberhalb des retinalen Pigmentepithels [RPE], die grünen Punkte die Flussregistrierung unterhalb des RPE).

Makulaödems (roter Bereich) und seine Beurteilung im Zusammenhang mit den korrespondierenden vaskulären Veränderungen. Für die OCTA-Analysen „codierte Retinatiefe“ bzw. „Retina“ wurde der Bereich der oberflächlichen und tiefen Retinaschichten gewählt, wie die im zugehörigen „B-Scan-Durchlauf“ aufgeführten Segmentierungslinien zeigen. In diesen Netzhautschichten können einige Mikroaneurysmen ebenso wie kleine nicht perfundierte Areale zuverlässig identifiziert werden. $\mathrm{Zu}$ dem zeigt sich die foveal-asvaskuläre Zone (FAZ) geringfügig vergrößert.

\section{Retinaler Venenverschluss (RVV)}

Auch die durch einen RVV hervorgerufenen ischämischen Bereiche sind in der OCTA gut darstellbar, wie die beiden nachfolgend vorgestellten Fälle eines Venenastverschlusses (VAV) exemplarisch zeigen. Dabei ist es mittels OCTA auch möglich, trotz des begrenzten Scanausschnitts von $6 \times 6 \mathrm{~mm}$ eine zusammenhängende Darstellung des retinalen Gefäßsystems bis in die mittlere Peripherie zu erstellen. So sind im Falle eines oberen hemisphärischen Venenverschlusses im Fundusbild in der Peripherie Blutungen und Cotton-Wool-Herde erkennbar, in der FA zeigen sich entsprechende ischämische Areale ( $\triangle$ Abb.4a,b). Auch mit der AngioPlex-OCTA lassen sich die nicht perfundierten Areale bis in die mittlere Peripherie zuverlässig darstellen, da es aufgrund der guten Kooperation des Patienten und der kurzen Aufnahmezeiten ohne Probleme möglich war, mehrere OCT-Aufnahmen auch außerhalb der Gefäßbögen zu erstellen und zu einem Bild zusammenzusetzen ( $\triangle$ Abb.4c). Insgesamt ergibt sich eine gute Übereinstimmung der Befunde aus FA und OCTA.

Dies zeigt auch der Vergleich von FA und OCTA im Falle eines superotemporalen nicht perfundierten VAV ( $\triangle \mathbf{A b b} .5 \mathbf{a}, \mathbf{b})$. Die in der FA sichtbaren nicht papillären Neovaskularisationen und Leckagen können in der AngioPlex-OCTA-Analyse „codierte Retinatiefe“ sofort den in Rot dargestellten, kleinen Neovaskularisationen zugeordnet werden. Deren Größe, Struktur und räumliche Ausdehnung sind in der OCTA im Gegensatz zur FA sehr detailliert erkennbar und lassen sich gut bewerten, da die Gefäßveränderungen nicht durch den Austritt von Fluoreszenzfarbstoff maskiert werden. Auch nicht perfundierte Areale werden mittels OCTA zuverlässig und in ihrer räumlichen Ausdehnung präzise erkannt und zeigen eine gute Übereinstimmung mit den Erkenntnissen aus der FA.

\section{Retinaler Arterienverschluss (RAV)}

Hinsichtlich RAV zeigt sich insgesamt eine sehr gute Übereinstimmung der Erkenntnisse aus FA und OCTA-Aufnahmen. Auch hier liefert die OCTA zusätzliche und wichtige tiefenselektive Informationen. Im Falle eines Patienten mit Verschluss der A. temporalis superior und gut sichtbarem Embolus im Fundusbild finden sich die in der FA dargestellten nicht perfundierten Areale in der OCTA-Aufnahme als massive Perfusionsstörungen im Verschlussgebiet wieder ( $\mathbf{A}$ bb. 6a-c). Im korrespondierenden „B-Scan-Durchlauf“ kann dies verifiziert werden, da im Bereich des Arterienverschlusses in den Netzhautschichten keinerlei Perfusion detektierbar ist ( Abb.6d, weiße Pfeile). Die verschlossene Arterie ist nicht mehr perfundiert und stellt sich somit auch nicht in der OCTA dar. Gerade bei älteren RAV lässt sich die Diagnose manchmal besser mit der OCTA sichern als mit der FA. 


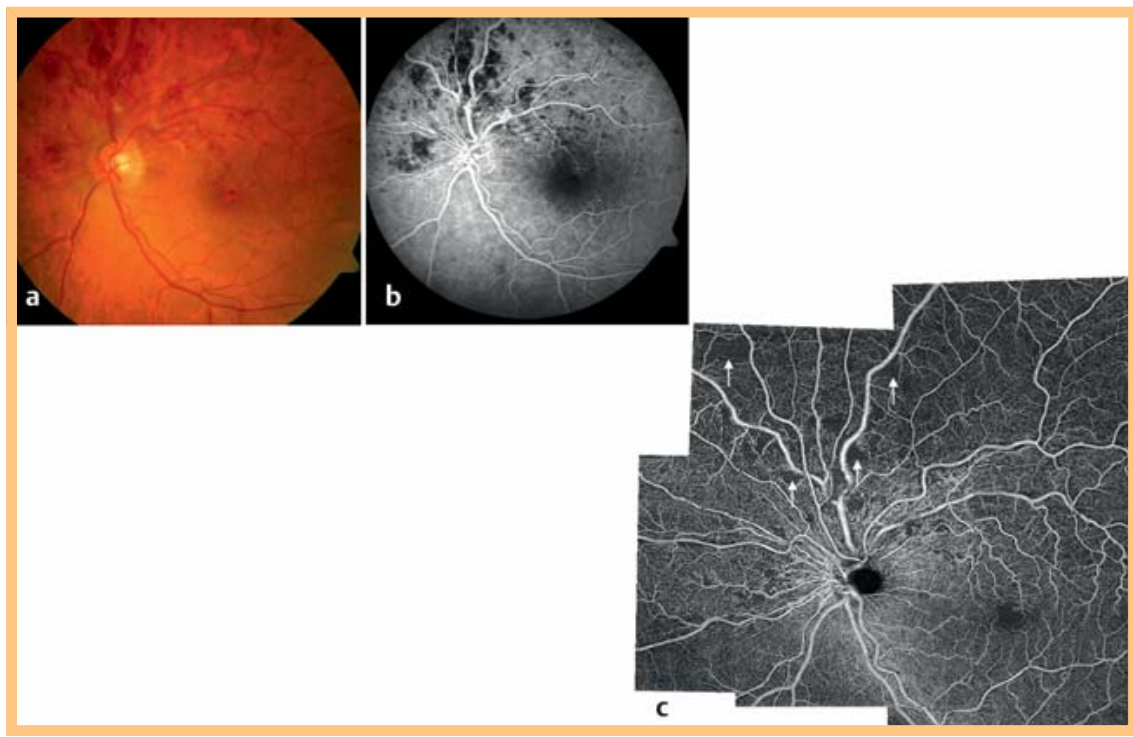

Abb. 4 Hemisphärischer Venenverschluss oben. a Das klinische Bild zeigt Blutungen und CottonWool-Herde; b Fluoreszenzangiografie; c OCTAngiografie-Darstellung der Retinagefäße mit nicht perfundierten Arealen (Pfeile).
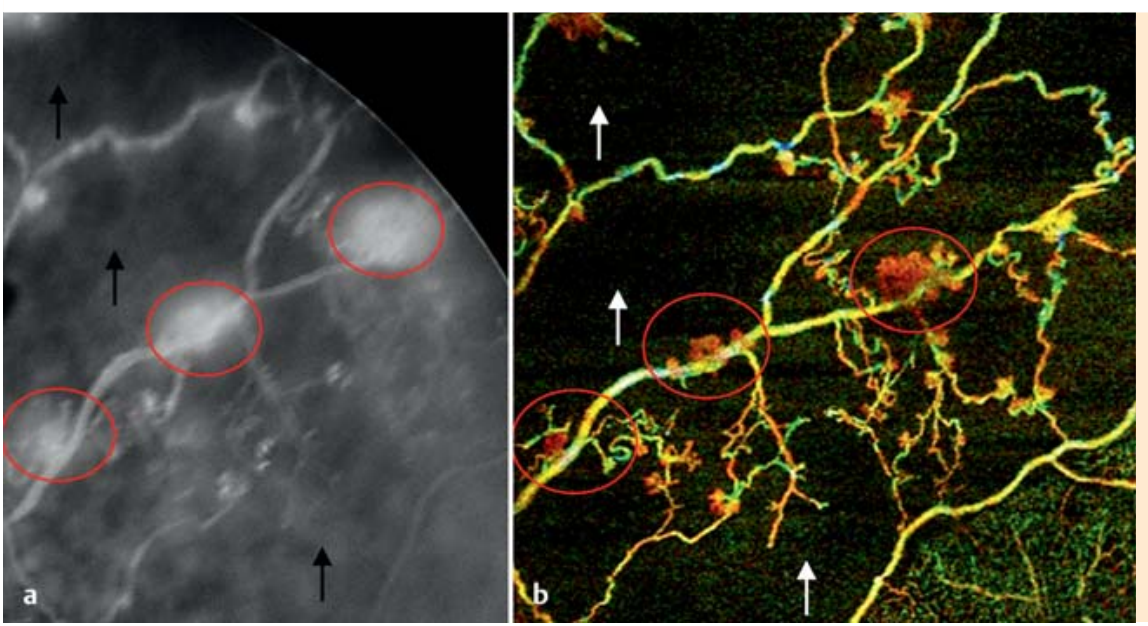

Abb. 5 Venenastverschluss mit nicht perfundierten Arealen (Pfeile) und präretinalen Neovaskularisationen (rote Kreise). a Fluoresceinangiografie; b codierte Retinatiefe OCT-Angiografie.

\section{Pathologische Myopie}

Im Falle einer pathologischen Myopie ermöglichte die AngioPlexOCTA im Rahmen einer Routineuntersuchung die frühzeitige Diagnose einer Neovaskularisation am asymptomatischen Partnerauge im Bereich eines umschriebenen extrafovealen Makulaödems, das sich in der Makuladickenmessung im OCT darstellte ( Abb.7a,b), sodass auf die Durchführung einer Fluoresceinangiografie verzichtet wurde. In der Analyse „Retina + OCT Makula“, in der die AngioPlex-Gefäßdarstellung mit der OCT-Makuladicke überlagert dargestellt wird, ist der Bereich des Makulaödems sowie eine darunter befindliche choroidale Neovaskularisation (CNV) sofort identifizierbar ( Abb. 7b). Die Segmentierung der inneren und tiefen Netzhautschichten zeigt eine präzise und detaillierte Darstellung der CNV ( $\bullet$ Abb. 7c). Zudem lässt sich im „B-Scan-Durchlauf“ die Lage der CNV sowie deren Perfusion bestätigen $(\bullet$ Abb. 7 d).

\section{Altersbezogene Makuladegeneration (AMD)}

Bei Patienten mit neovaskulärer AMD ergibt sich mit OCTAUntersuchungen bislang ein komplexes Bild, was angesichts der vielfältigen Ausprägungen choroidaler Neovaskularisationen in Bezug auf Lage und Ausdehnung der CNV auch zu erwarten war. Sowohl in der FA als auch in der OCTA detektierbare CNV ermög- lichen Vergleiche zwischen den Ergebnissen der beiden Diagnosemethoden. Exemplarisch sind hier die Aufnahmen einer vorwiegend klassischen CNV mit umgebenden Anteilen einer okkulten CNV dargestellt ( $\bullet$ Abb. 8a-f). Die klassischen Anteile der CNV sind in der FA als klar abgegrenzte Hyperfluoreszenz in der Frühphase und als scharf begrenzte, zunehmende Leckage in der Spätphase identifizierbar, wobei durch den Farbstoffaustritt die Gefäße maskiert sind und daher keine wesentlichen Informationen zur Gefäßstruktur gewonnen werden können ( $\bullet$ Abb. 8b,c). In der korrespondierenden OCTA-Analyse „codierte Retinatiefe“ sind die entsprechenden klassischen Anteile der CNV als blau eingefärbte Gefäße sofort gut erkennbar ( $\triangle$ Abb. 8d). Die anschließende Segmentierung im Bereich der CNV liefert ein sehr detailreiches Bild der klar abgegrenzten CNV-Anteile oberhalb des retinalen Pigmentepithels (RPE; Typ-2-CNV) und unter dem RPE (Typ-1-CNV; $\bigcirc$ Abb.8 e, $\bigcirc$ Video 1). Zudem kann im „BScan-Durchlauf" die Perfusion der CNV verifiziert werden ( Abb. 8f). Somit liefert auch hier die OCTA zur FA zusätzliche, detaillierte und tiefenspezifische Informationen und ermöglicht es, Perfusionsstatus, Ausmaß und Lage der Neovaskularisationen in Bezug auf die Lage in den verschiedenen Schichten 3-dimensional präzise zu bestimmen. 

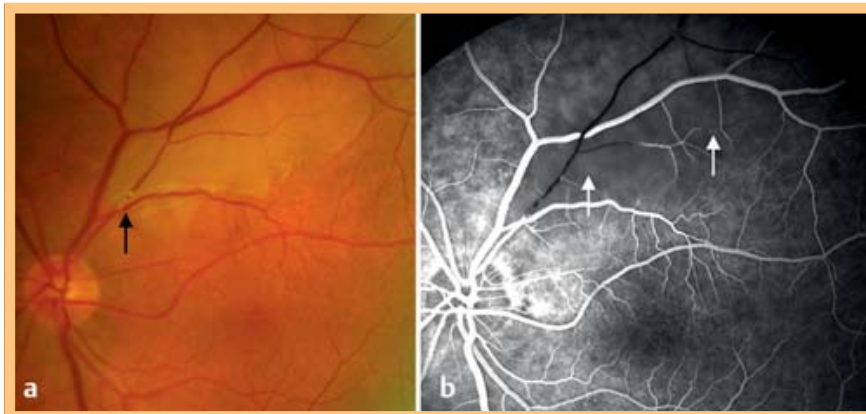

Abb. 6 Retinaler Arterienverschluss der A. temporalis superior. a Klinisches Bild mit sichtbarem Embolus (Pfeil) und Netzhautödem; b fluoreszenzangiografischer Befund mit nicht perfundierten Arealen (Pfeile); c Die OCT-Angiografie zeigt korrespondierend zur Fluoreszenzangiografie massive Perfusions-

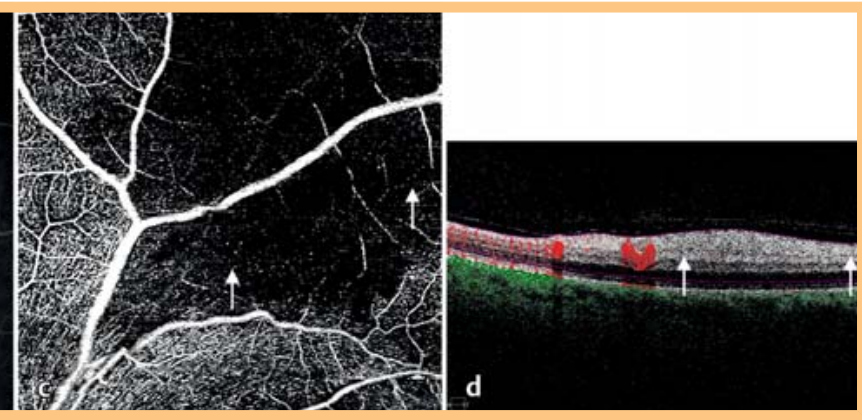

störungen (Pfeile) im Verschlussgebiet; d B-Scan-Durchlauf: rote Punkte zeigen die Flussregistrierung der Netzhaut, grüne Punkte die der Aderhaut, der Bereich des Arterienverschlusses weist keine Perfusion mehr auf (Pfeile).
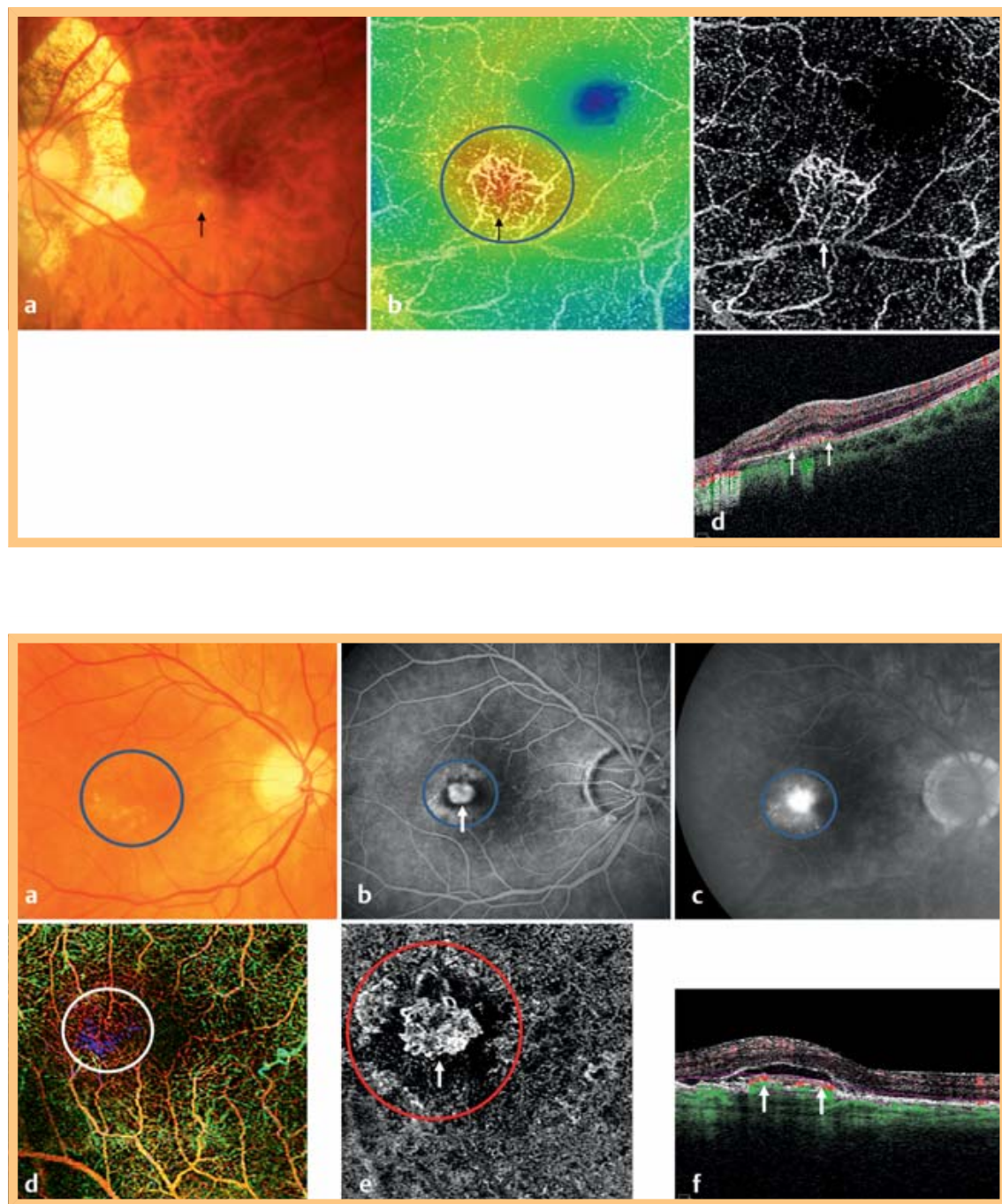

Abb. 7 Pathologische Myopie. a Das klinische Bild zeigt ein Makulaödem (Pfeil); b Die OCT-Angiografie-Gefäßdarstellung überlagert mit OCT-Makuladicke zeigt die choroidale Neovaskularisation (Pfeil) mit überlagertem OCT der Makula mit Ödem (Kreis); c choroidale Neovaskularisation (Pfeil); d B-Scan-Durchlauf mit Segmentierungslinien (lila), rote Punkte zeigen die Flussregistrierung der Netzhaut, grüne Punkte die der Aderhaut, CNV-Bereich siehe Pfeile.

Abb. 8 Neovaskuläre altersbezogene Makuladegeneration. a Das klinische Bild zeigt ein Makulaödem (Kreis); b choroidale Neovaskularisation: Fluoresceinangiografie Frühphase (CNV durch Kreis markiert), mit zentralem, klassischem Anteil der CNV (Pfeil) und c Spätphase (CNV durch Kreis markiert); d CNV in codierter Retinatiefendarstellung in Blau (weißer Kreis); e choroidale Neovaskularisation (roter Kreis) in der OCT-Angiografie mit CNVAnteil korrespondierend zu klassischer CNV in Bild b (Pfeil); f Makulaödem im B-Scan-Durchlauf mit Segmentierungslinien (lila) im Bereich der CNV, rote Punkte zeigen die Flussregistrierung der Netzhaut, grüne Bereiche die der Aderhaut an (CNV siehe Pfeile).

\section{Diskussion}

\section{$\nabla$}

Die OCT-Angiografie stellt ein hochinteressantes, neues Bildgebungsverfahren dar, mit dem in nur wenigen Sekunden und nicht invasiv das Mikrogefäßsystem der Netzhaut und der Aderhaut über Flussregistrierung erfasst und als hochaufgelöstes, 3dimensionales Bild dargestellt werden kann. Dabei liefert die OCTA gleichzeitig Daten sowohl zum Perfusionsstatus als auch zur morphologischen Struktur und erleichtert so die präzise Diagnosestellung zahlreicher Erkrankungen des Hinterabschnitts. Wesentlichster Vorteil der neuen Bildgebung ist ihre 3-dimensionale Visualisierung der Gefäße in hoher Auflösung, wohingegen in der FA sämtliche Informationen in 2 Dimensionen zusammengefasst dargestellt werden. So liefert die OCTA zusätzlich zur FA detailreiche Tiefeninformationen und ermöglicht eine deutlich präzisere Beurteilung des räumlichen Verlaufs der Ge- 


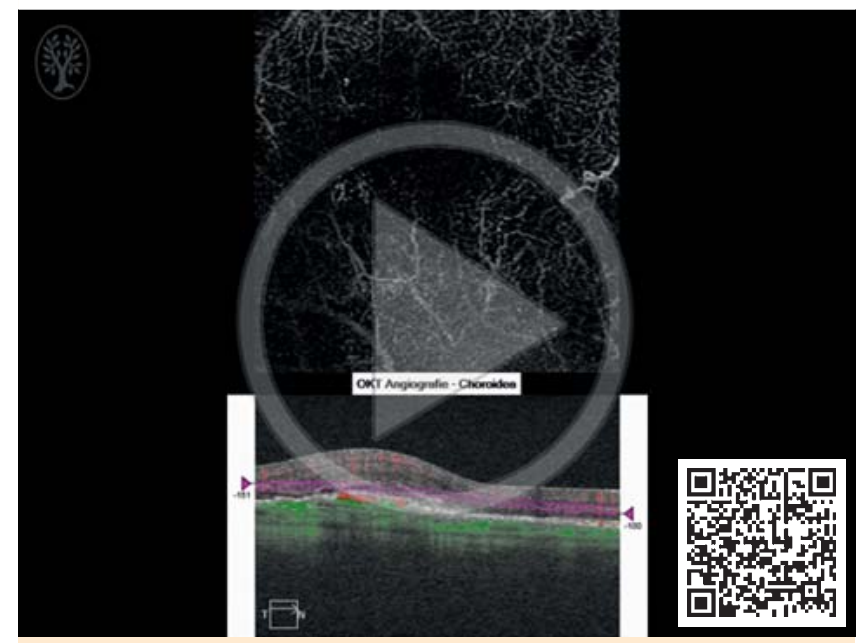

Video 1 Video zur OCTA in $\bullet$ Abb. 8 e, f zeigt klassischen CNV-Anteil oberhalb des RPE und Typ-1-CNV-Anteil unter dem RPE. http://tinyurl.com/KliMo-05-16-Lang

fäße bei unterschiedlichen Erkrankungen des Hinterabschnitts. Darüber hinaus erfordert die OCTA im Gegensatz zur FA keine mit den bekannten Nebenwirkungen, Risiken und Kontraindikationen einhergehende Farbstoffinjektion. Daher kann die OCTA problemlos häufiger und ohne wesentliche Belastungen für den Patienten und auch ohne Pupillenerweiterung durchgeführt werden. Sie ist auch aufgrund ihrer einfachen und schnellen Durchführung sehr gut als Monitoring- bzw. Screening-Methode geeignet und kann so frühzeitige Diagnosestellungen ermöglichen. Allerdings können mit der OCTA nur perfundierte Bereiche und Veränderungen dargestellt werden. Sie liefert auch keine direkten Hinweise über Schrankenstörungen der Gefäße. Dies ist lediglich indirekt durch die Überlagerung der OCTA-Befunde mit der Makuladickenmessung möglich. Ein Vorteil der Fluoreszenzangiografie ist der deutlich größere Bildausschnitt, der insbesondere mit den Weitwinkelkameras eine Darstellung der Netzhaut bis in die Peripherie ermöglicht und auch eine bessere Übersicht über die Lage der Veränderung liefert.

Erste klinische Erfahrungen zeigen, dass sich die OCT-Angiografie gut in die Standarduntersuchungen zur Diagnose bzw. zum Monitoring von Erkrankungen der Retina und Aderhaut integrieren lässt, da sie einfach anwendbar und schnell durchführbar ist. Aufgrund der „Single-Scan Simplicity“ der AngioPlex-OCTA-Technologie werden die OCTA-Aufnahmen mit nur einem zusätzlichen Scan bereitgestellt. Dies trägt gemeinsam mit kurzen Scanaufnahmezeiten (5-10 Sekunden pro Scan) und den übersichtlich auf dem Gerätedisplay angeordneten Datenanalysen zu schnellen Arbeitsabläufen und über voreingestellte Algorithmen zur ausführlichen Befundung bei. Die hohe Bildqualität sowie die Farbcodierung bestimmter Netzhautschichten erleichtert eine schnelle Orientierung in der Tiefe und die „Film-Option“ ermöglicht eine schnelle Identifizierung klinisch relevanter Netzhautschichten. Die kombinierten Analysedarstellungen und die Möglichkeit, auch intensitätsbasierte SD-OCT-Daten aufzurufen und gemeinsam mit den Perfusionsdaten zu beurteilen, erleichtern die Befundung. Insgesamt liefert die OCT-Angiografie eine schnelle, komfortable und sehr präzise 3-dimensionale Visualisierung der Mikrogefäßsysteme von Retina und Aderhaut in beliebigen Schichten.

\section{Retinale vaskuläre Erkrankungen}

Mittels OCTA können Gefäßveränderungen wie Mikroaneurysmen, mikrovaskuläre Anomalien, Neovaskularisationen und nicht perfundierte Bereiche mit einem bislang nicht erreichten Detailreichtum hinsichtlich ihrer Lage und Struktur dargestellt werden. Insbesondere bei Erkrankungen mit diesen Gefäßanomalien kann die OCTA daher zusätzliche und unverzichtbare Information zur Diagnose, während der Therapie und zur Beobachtung des Krankheitsverlaufs liefern. Mittels OCTA kann eine zusammenhängende Darstellung des retinalen Gefäßsystems bis in die mittlere Peripherie über Aufnahmen in den verschiedenen Netzhautbereichen erzielt werden.

Auch wenn Leckagen aus den Gefäßen in der OCTA nicht darstellbar sind, können mit der OCT-Dickenmessung jedoch Makulaödeme (MÖ) erfasst, quantifiziert und dem OCTA-Bild überlagert werden, sodass eine Diagnose von MÖ mittels OCTA und korrespondierenden SD-OCT-Untersuchungen zuverlässig möglich ist. Die OCTA liefert zahlreiche wichtige zusätzliche Informationen (detaillierte, hochaufgelöste Darstellung/Tiefeninformation) zur FA, die für eine präzise Diagnosestellung, Therapie und Verlaufskontrolle unverzichtbar sind.

Bezüglich DR konnten alle mikrovaskulären Veränderungen wie Mikroaneurysmen, intraretinale mikrovaskuläre Anomalien, nicht perfundierte Areale, Vergrößerung der FAZ und Neovaskularisationen gut dargestellt werden. Takase et al. [7] konnten mittels OCTA nachweisen, dass die FAZ bei Diabetikern sowohl mit, aber auch ohne Vorhandensein von diabetischer Retinopathie vergrößert ist.

Auch hinsichtlich RVV konnten wir eine gute Übereinstimmung der Erkenntnisse aus FA und OCTA-Aufnahmen feststellen. Rispoli et al. [19] fanden bei 10 Patienten mit VAV mittels OCTA eine Verringerung der Kapillardichte, FAZ-Vergrößerung und nicht perfundierte kapilläre Areale in den oberflächlichen und tiefen Netzhautgefäßschichten.

Hinsichtlich RAV zeigt sich eine exzellente Übereinstimmung von FA und OCTA. Ein RAV kann anhand von klinischen Bildern und OCTA-Aufnahmen präzise diagnostiziert werden. Bonini et al. [12] konnten in 86\% die Perfusionsstörungen im oberflächlichen und tiefen retinalen Kapillarplexus korrespondierend zur FA nachweisen.

\section{Altersbezogene Makuladegeneration}

Bezüglich AMD stellt die OCTA eine neue Methode mit einem sehr großen Potenzial hinsichtlich Diagnose, Verlaufskontrollen, Erfassung von Rezidiven und rechtzeitiger Therapie dar. Da in der OCTA die Einzelgefäße der CNV nicht wie in der FA bei okkulter CNV oder durch Farbstoffleckage maskiert sind, ermöglicht die OCTA eine sehr exakte Detektion der Gefäßstruktur und des Perfusionsstatus der CNV. Zudem besteht mittels OCTA die Möglichkeit, die Lage der Neovaskularisationen in Bezug zum RPE zu bestimmen und zwischen drusenbedingten und mit Gefäßneubildung assoziierten Pigmentepithelabhebungen zu differenzieren. Insbesondere im „B-Scan-Durchlauf“ lässt sich gut erkennen, ob die Pigmentepithelabhebung vaskularisiert ist oder nicht. Diese Unterscheidung ist klinisch von großer Bedeutung, da die Vaskularisierung einer Pigmentepithelabhebung das Vorliegen einer neovaskulären, behandlungsbedürftigen Form der AMD darstellt $[20,21]$. So konnten Roisman et al. in 3 von 11 Augen mit asymptomatischer, intermediärer AMD, die noch nicht exsudativ waren, in der OCTA bereits eine Typ-1-CNV nachweisen [22]. Hier könnte die OCTA aufgrund ihres nicht invasiven Charakters und ihrer - im Gegensatz zur Fluoreszenzangiografie - einfachen 
und schnellen Durchführung bereits zu einem sehr frühen Zeitpunkt der AMD-Erkrankung zu regelmäßigen Kontrolluntersuchungen eingesetzt werden, um möglichst frühzeitig Zeichen einer Krankheitsprogression wie Entstehung oder Reaktivierung der Neovaskularisationen zu entdecken und rechtzeitig zu behandeln. Selbstverständlich müssen zu diesem Zweck im Rahmen prospektiver, klinischer Studien zunächst exakte Befunde erhoben und einheitliche Analysestrategien sowie neue AMDKlassifizierungen basierend auf OCTA-Untersuchungen definiert und evaluiert werden. Allererste Untersuchungen dazu liegen bereits vor $[4,13-16,22]$.

\section{Fazit \\ $\nabla$}

Zusammenfassend lässt sich somit festhalten, dass die OCTA eine äußerst vielversprechende, neue Diagnosemethode darstellt, die mit hoher Wahrscheinlichkeit zu einer Revolution der Diagnostik von Netz- und Aderhauterkrankungen führen wird. Mit der OCTA kann in nur wenigen Sekunden und nicht invasiv ein hochaufgelöstes, 3-dimensionales Bild des Mikrogefäßsystems von Netzhaut und Aderhaut erfasst werden. Selbst kleinste Veränderungen perfundierter retinaler und choroidaler Gefäße können ohne Kontrastmittelinjektionen präzise und tiefenselektiv dargestellt werden, sodass die OCTA wichtige zusätzliche Informationen zur FA liefert. Die OCTA lässt sich leicht in den Klinikalltag integrieren und ihre nicht invasive, schnelle Durchführung trägt dazu bei, pathologische Gefäßveränderungen identifizieren, klassifizieren und über die Zeit verfolgen zu können, woraus sich Möglichkeiten eines verbesserten Monitorings ergeben. Dazu wird es erforderlich sein, auf OCTA-Untersuchungen basierende Klassifizierungen und einheitliche Analysestrategien für verschiedene Erkrankungen zu definieren und im Rahmen prospektiver, klinischer Studien zu evaluieren. Die OCTA stellt eine Untersuchungsmethode dar, auf die bereits jetzt nicht mehr verzichtet werden kann. Ob sie die Fluoreszenzangiografie weitgehend ersetzen wird, lässt sich jedoch derzeit noch nicht endgültig absehen.

\section{Interessenkonflikt}

Finanzielle Unterstützung, auch für die OpenAccess-Publikationsgebühr, durch die Firma Carl Zeiss Meditec Vertriebsgesellschaft mbH, Oberkochen.

\section{Literatur}

1 Huang Y, Zhang $Q$ Thorell MR et al. Swept-source OCT angiography of the retinal vasculature using intensity differentiation-based optical microangiography algorithms. Ophthalmic Surg Lasers Imaging Retina 2014; 45: 382-389

2 Spaide RF, Klancnik JM, Cooney MJ et al. Retinal vascular layers imaged by fluorescein angiography and optical coherence tomography angiography. JAMA Ophthalmol 2015; 133: 45-50

3 Matsunaga D, Yi J, Puliafito CA et al. OCT angiography in healthy human subjects. Ophthalmic Surg Lasers Imaging Retina 2014; 45: 510-515

4 Jia Y, Bailey ST, Wilson DJ et al. Quantitative optical coherence tomography angiography of choroidal neovascularization in age-related macular degeneration. Ophthalmology 2014; 121: 1435-1444

5 Wang X, Jia Y, Spain R et al. Optical coherence tomography angiography of optic nerve head and parafovea in multiple sclerosis. $\mathrm{Br} \mathrm{J}$ Ophthalmol 2014; 98: 1368-1373

6 Freiberg FJ, Pfau M, Wons J et al. Optical coherence tomography angiography of the foveal avascular zone in diabetic retinopathy. Graefes Arch Clin Exp Ophthalmol 2015 [Epub ahead of print]

7 Takase N, Nozaki M, Kato A et al. Enlargement of foveal avascular zone in diabetic eyes evaluated by en face optical coherence tomography angiography. Retina 2015; 35: 2377-2383

8 Thorell MR, Zhang $Q$ Huang Y et al. Swept-source OCT angiography of macular telangiectasia type 2. Ophthalmic Surg Lasers Imaging Retina 2014; 45: 369-380

9 Schwartz DM, Fingler J, Kim DY et al. Phase-variance optical coherence tomography: a technique for noninvasive angiography. Ophthalmology $2014 ; 121: 180-187$

10 Jia $Y$, Wei E, Wang $X$ et al. Optical coherence tomography angiography of optic disc perfusion in glaucoma. Ophthalmology 2014; 121: 1322 1332

11 Costanzo E, Cohen SY, Miere A et al. Optical coherence tomography angiography in central serous chorioretinopathy. J Ophthalmol 2015; 2015: 134783

12 Bonini Filho MA, de Carlo TE, Ferrara D et al. Association of choroidal neovascularization and central serous chorioretinopathy with optical coherence tomography angiography. JAMA Ophthalmol 2015; 133 : 899-906

13 Pauleikoff D, Heimes B, Spital G et al. OCT-Angiografie - ist dies die Zukunft der makulären Diagnostik? Klin Monatsbl Augenheilkd 2015; 232: 1069-1076

14 Fang PP, Lindner M, Steinberg JS et al. Klinische Anwendungen der OCTAngiographie. Ophthalmologe 2016; 113: 14-22

15 Lommatzsch A, Farecki ML, Book B et al. OCT-Angiographie bei der exsudativen altersbedingten Makuladegeneration - Erste Erfahrungen. Ophthalmologe 2016; 113: 23-29

16 De Carlo TE, Bonini MA, Chin AT et al. Spectral-domain optical coherence tomography angiography of choroidal neovascularization. Ophthalmology 2015; 122: 1228-1238

17 Kuehlewein L, Bansal M, Lenis TL et al. Optical coherence tomography angiography of type 1 neovascularization in age-related macular degeneration. Am J Ophthalmol 2015; 160: 739-748.e2

18 Zhang A, Zhang $Q$ Chen CL et al. Methods and algorithms for optical coherence tomography-based angiography: a review and comparison. J Biomed Opt 2015; 20: 100901

19 Rispoli M, Savastano MC, Lumbroso B. Capillary network anomalies in branch retinal vein occlusion on optical coherence tomography angiography. Retina 2015; 35: 2332-2338

20 Hartnett ME, Weiter JJ, Garsd A et al. Classification of retinal pigment epithelial detachments associated with drusen. Graefes Arch Clin Exp Ophthalmol 1992; 230: 11-19

21 Pauleikhoff D, Löffert D, Spital G et al. Pigment detachment in the elderly. Clinical differentiation, natural course, and pathogenic implications. Graefes Arch Clin Exp Ophthalmol 2002; 240: 533-538

22 Roisman L, Zhang $Q$ Wang RK et al. Optical coherence tomography angiography of asymptomatic neovascularization in intermediate agerelated macular degeneration. Ophthalmology 2016; DOI: 10.1016/j. ophtha.2016.01.044 [Epub ahead of print] 